\title{
Discovery of X-ray flaring on the magnetic Bp-star $\sigma$ Ori E
}

\author{
D. Groote and J. H. M. M. Schmitt
}

\author{
Hamburger Sternwarte, Gojenbergsweg 112, 21029 Hamburg, Germany
}

Received 10 September 2003 / Accepted 7 January 2004

\begin{abstract}
We report the detection of an X-ray flare on the Bp star $\sigma$ Ori E with the ROSAT high resolution imager (HRI). The flare is shown to have likely occurred on the early-type star, rather than on an hypothesized late-type companion. We derive flare parameters such as total energy release, coarse estimates of size and density, and also present arguments for a magnetic origin of the flare. We place our observations in the context of a magnetic character of Bp-type stars and speculate on a common physical basis and connection between $\mathrm{Bp}$ and Be stars.
\end{abstract}

Key words. stars: chemically peculiar - stars: early-type - stars: flare - stars: individual: HD 37479, $\sigma$ Ori E stars: magnetic fields - stars: winds, outflows

\section{Introduction}

For cool stars with outer convective envelopes, X-ray emission is generally considered a proxy indicator of magnetic activity. Systematic surveys of X-ray emission among cool stars in the solar neighborhood have shown the occurrence of X-ray emission for all stars of spectral type $\mathrm{F}$ through $\mathrm{M}$ on the main sequence (Schmitt et al. 1995; Schmitt 1997; Schmitt \& Liefke 2004). The observed X-ray luminosities vary (from star to star) over almost four orders of magnitude, and the most important parameter governing the X-ray output level of a given cool star appears to be its rotation rate (Pallavicini et al. 1981). The X-ray emission from cool stars is (often) time-variable. Specifically, X-ray flaring is frequently observed among cool stars, and in particular, X-ray flaring has been observed for all types of cool stars. Such X-ray flaring is readily explained by the sudden release of magnetic energy and its ultimate conversion into heat and radiation. The X-ray properties of cool stars have to be contrasted to the X-ray properties of hot stars without outer convective envelopes. To avoid complications from the possibility of X-ray emission from interacting winds in O-star binary systems, we here restrict our attention to single stars. Similarly to cool stars, all single hot stars appear to be $\mathrm{X}$-ray emitters, at least down to a spectral type of around B2. In contrast to cool stars, the X-ray luminosity of hot stars appears to be independent of rotation; rather their X-ray emission is characterized by the relation $L_{\mathrm{X}} / L_{\mathrm{bol}} \approx 10^{-7}$ (cf., Pallavicini et al. 1981). Thus hot stars can be very strong X-ray sources in terms of their total X-ray luminosity $L_{\mathrm{X}}$, however, in comparison to their total radiative output only small fractions of their total luminosity are radiated at X-ray energies.

Send offprint requests to: D. Groote, e-mail: dgroote@hs.uni-hamburg.de
The X-ray emission from hot stars is usually attributed to instabilities in their radiatively driven winds, leading to stochastic velocity fields and shocks (cf., Lucy 1982). Surprisingly, despite the stochastic nature of the X-ray production process in hot stars, their X-ray emission level appears to be very stable (cf., Berghöfer \& Schmitt 1995, 1994) and convincing reports of variable X-ray emission from early type stars are rare. For example, Berghöfer \& Schmitt (1994) report long-term spectral variability in the hot supergiant $\zeta$ Ori, which they interpret as a propagating shock wave, and Gagné et al. (1997) report a periodic variation of the X-ray flux of $\theta^{1}$ Ori C, which appears to be modulated with the star's rotation period of 15.4 days. An actual X-ray flare in the B2e star $\lambda$ Eri has been reported by Smith et al. (1993); an increase in count rate by about a factor of 6 over quiescent values over about $24 \mathrm{~h}$ was observed leading to an overall energy release of $2 \times 10^{36} \mathrm{erg}$.

In general, magnetic fields are thought to be unimportant for the dynamics of the outer envelopes of hot stars. While there are no reasons not to assume the presence of magnetic fields on such stars, the typically available magnetic field strength upper limits of a few hundred Gauss show that the magnetic field pressure is much smaller than the ram pressures of their winds (Linsky 1985). Successful measurements (rather than upper limits) of magnetic fields in early-type stars are rare. One of the few examples is $\theta^{1}$ Ori $\mathrm{C}$, where a magnetic field of about $1.1 \mathrm{kG}$ has been detected to be modulated with the rotation period of 15.4 days (Donati et al. 2002). The prototypical He-strong He-variable star $\sigma$ Ori E (HD 37479; spectral type B2Vp) is known to have a strong magnetic field of about $10 \mathrm{kG}$ polar field strength (Landstreet \& Borra 1978). A weak stellar wind of some $10^{-10} M_{\odot} \mathrm{y}^{-1}$ is channeled along magnetic field lines. In closed magnetic loops matter is captured, forming torus-shaped clouds (or a ring) in the plane of the 
magnetic equator (Groote \& Hunger 1982 (GH1); Shore \& Brown 1990). These clouds are magnetically coupled to the star, thus corotate and occult the star twice during one rotational period leading to absorption in the Stroemgren $u$-band (Hesser et al. 1977), the higher Balmer lines (Groote \& Hunger 1976), and in the strong resonance UV lines of CIV, SiIV (Smith \& Groote 2001). When viewed face on, the clouds exhibit redshifted emission in $\mathrm{H}_{\alpha}$ and some of the above metal lines. The clouds extend to about $6 R_{*}$ and are believed to release matter at their inner boundary back to the stellar surface, thus increasing the $\mathrm{He}$ abundance near the magnetic equator (Groote 2003). First results from 2D MHD simulations by udDoula \& Owocki (2002) support this scenario.

Observations of $\sigma$ Ori E at radio wavelengths $(6 \mathrm{~cm}$, Drake et al. 1987) are consistent with the assumption of synchrotron radiation by mildly relativistic electrons. X-rays from $\sigma$ Ori $\mathrm{E}$ have been observed by Berghöfer \& Schmitt (1994) at a constant level of about $8.2 \times 10^{-3}$ counts s $^{-1}$ using the ROSAT high resolution imager (HRI). The origin of this X-ray emission is unclear.

Converting the observed $\mathrm{X}$-ray count rate to an X-ray flux yields a spectrum-dependent estimate of approximately $2 \times 10^{-13} \mathrm{erg} \mathrm{cm}^{-2} \mathrm{~s}^{-1}$ (appropriate for $T \approx 20 \mathrm{MK}$ and $N_{\mathrm{H}} \approx$ $10^{21} \mathrm{~cm}^{-2}$ ), which corresponds to a fraction $\approx 3.9 \times 10^{-7}$ of the total bolometric flux of $\sigma$ OriE. $\sigma$ Ori E thus lies in line with the typical values observed from O-type stars. Alternatively, the magnetic geometry of $\sigma$ Ori E might be relevant and the X-ray emission might originate from frictional heating in the wind when helium and hydrogen decouple from the driving metals (Krtička \& Kubát 2001) and/or from the impact of wind particles into the ring/clouds (Babel \& Montmerle 1997a). Since the above described ring is constantly filled with new material from the star, matter also has to be released and it is natural to assume magnetic field line reconnection with heating to some $10^{6} \mathrm{~K}$ in analogy to the Earth's magnetosphere. This release of matter is not expected to occur continuously, but rather sporadically, leading to variable $\mathrm{X}$-ray emission.

However, in contrast to these expectations, first observations (duration about $15 \mathrm{ks}$ ) with the ROSAT HRI (Berghöfer \& Schmitt 1994, 1995) did not show any significant temporal variations in the X-ray flux from $\sigma$ Ori E. Since then more data on $\sigma$ Ori $\mathrm{E}$ (about $75 \mathrm{ks}$ distributed over more than 30 days) were taken with the ROSAT HRI within the context of another program and it is the purpose of this paper to present (Sect. 2) and discuss these observations (Sect. 3). We determine basic flare properties and will in particular compare the $\sigma$ Ori E data with the flare found in $\lambda$ Eri (Smith et al. 1993). Our conclusions will be drawn in Sect.4, and finally we speculate on a possible connection between $\mathrm{Bp}$ and Be stars.

\section{Observations and data analysis}

A series of pointings with the ROSAT HRI was carried out on the multiple O-type system $\sigma$ Ori AB. The ROSAT HRI detector has very little intrinsic energy resolution, but very good angular resolution $(\approx 5 \operatorname{arcsec})$. A separation of the X-ray emission from $\sigma$ Ori $\mathrm{AB}$ and $\sigma$ Ori $\mathrm{E}$ was therefore straightforward, but no information on the shapes of the X-ray energy

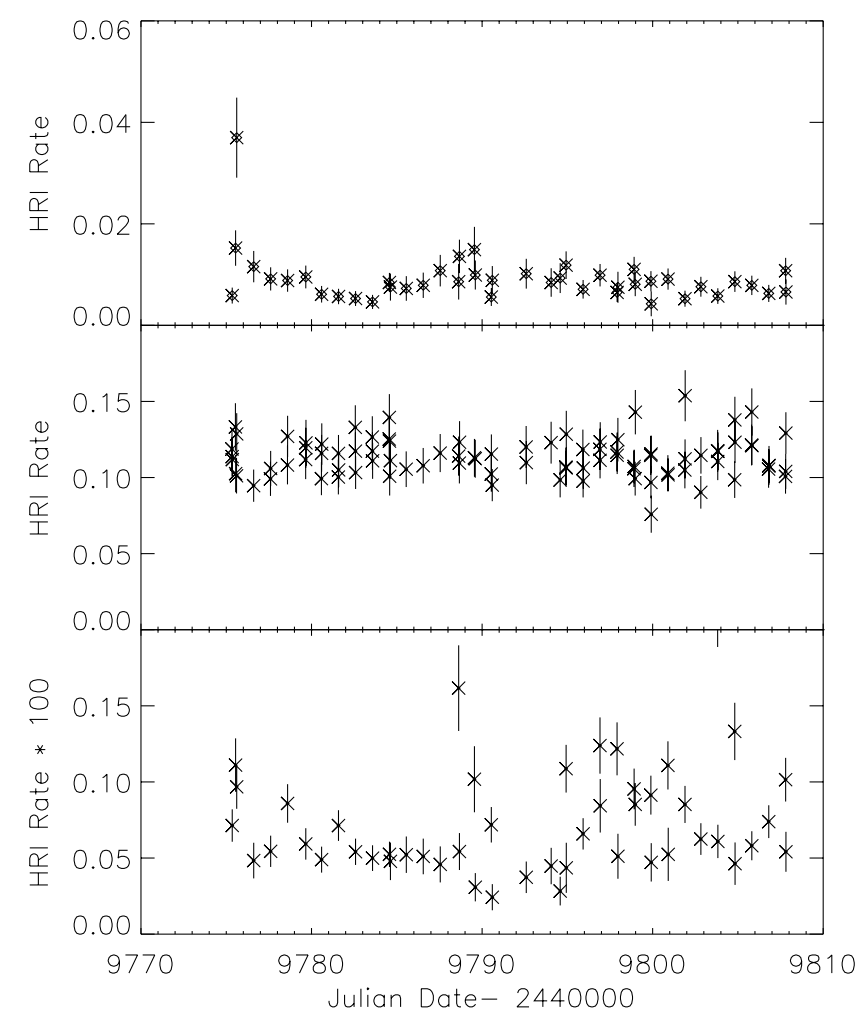

Fig. 1. ROSAT HRI light curves of $\sigma$ Ori E (top panel), $\sigma$ Ori AB (medium panel), and a representative background region (bottom panel); note that the background rate has been increased by a factor of 100 for better visibility. Note also that background contributions to the source count rates are very small.

distributions of $\sigma$ Ori $\mathrm{AB}$ and $\sigma$ OriE was available. The ROSAT observations were carried out as a sequence of individual snapshots, each lasting approximately $2000 \mathrm{~s}$, separated by approximately $24 \mathrm{~h}$. The total time span of the X-ray observations covered 30 days from JD $2449775-J D 2449808$, and the total source integration time was about $74 \mathrm{ks}$.

The data analysis was carried out within the EXSAS content in the MIDAS environment; the ROSAT sequences analyzed were the ROR numbers 201 882-201915. The photon event tables and event rates files from the sequences were merged with the EXSAS command MERGE into one effective observation and an image containing the data in the pulse height channels 2-9 was created. From this image the photons originating from $\sigma$ Ori $\mathrm{E}$ and $\sigma$ Ori $\mathrm{AB}$ as well as a background region were extracted; for the light curves we used a circular extraction region of 13 arcsec, while for the background a much larger region in the central part of the detector was chosen. From the source photon files light curves were generated with corrections for dead time and vignetting; note that these latter corrections are very small and are far below the statistical errors in each time bin.

\subsection{X-ray light curves}

The generated, but not background subtracted, light curves for $\sigma$ Ori E, $\sigma$ Ori AB and the background are shown in Fig. 1 as count rate versus modified Julian date. Note that the 


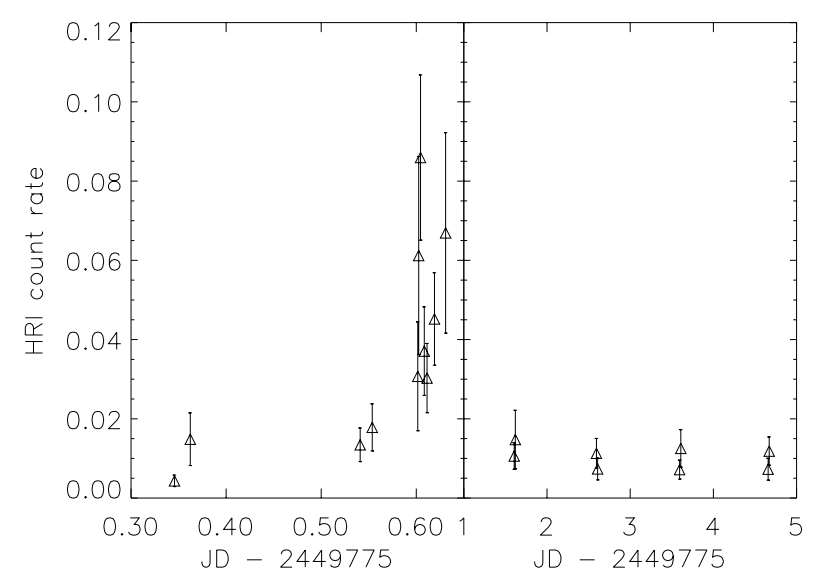

Fig. 2. ROSAT HRI light curve of $\sigma$ Ori E during flare increase (left panel) and constant X-ray fluxes on following observations (right panel). Note the different time scales and the jump between the two panels.

background count rate was very low, about a factor 100 below that of $\sigma$ Ori $\mathrm{E}$ and about a factor 400 below that of $\sigma$ Ori AB; although the background count rate does vary, its variations do not influence the source light curves. As is clear from Fig. 1, the light curve for $\sigma$ Ori AB stays constant, while in the case of $\sigma$ Ori $\mathrm{E}$ the first data points are low and comparable to the X-ray emission level observed by Berghöfer et al. (1994). Then the count rate increases rapidly over the next data bins, and returns to the pre-flare level with possibly some small variations. In Fig. 2 we show an enlargement of the X-ray light curve during Julian day 2449775 . The increase in X-ray count rate took place on a time scale of at most $2.5 \mathrm{~h}$. Whether our observations cover the peak of the flaring emission is unclear; $24 \mathrm{~h}$ later, at JD $=2440776.55$, when the next ROSAT observations were taken, the emission was back at pre-flare levels. It is very instructive to over-plot the ROSAT X-ray light curve of $\sigma$ Ori $E$ with the ROSAT PSPC light curve of $\lambda$ Eri reported by Smith et al. (1993; diamonds in Fig. 2); the $\lambda$ Eri (PSPC) data were appropriately shifted in time and scaled to the same apparent pre- and post flare levels. As is obvious from Fig. 2, the $\sigma$ OriE flare light curve is consistent with the observed light curve morphology of $\lambda$ Eri.

The statistical significance of the observed count rate increase in $\sigma$ Ori $\mathrm{E}$ is extremely high. If we assume a quiescent rate of 0.008 (HRI) cts s ${ }^{-1}$ (which may actually be a little higher than observed), we expect 23.1 photons in the $2886.5 \mathrm{~s}$ time interval between JD 2449775.563 and JD 2449775.631. This has to be contrasted with the total number of 82 photons, that were recorded during that time interval. Assuming Poisson statistics, the probability to observe 82 photons with $23.1 \mathrm{ex}$ pected is almost zero. In Fig. 2 one recognizes a more or less linear increase with two data points (at JD 2449775.65 ) above the linear curve. We analyzed - using a Kolmogorov-Smirnov test - the individual photon arrival times during this short data interval and found that deviations from constancy are possible at the $\approx 95 \%$ confidence limit. Thus shorter time scale variability is possible but not necessarily required by our HRI observations of $\sigma$ Ori E.

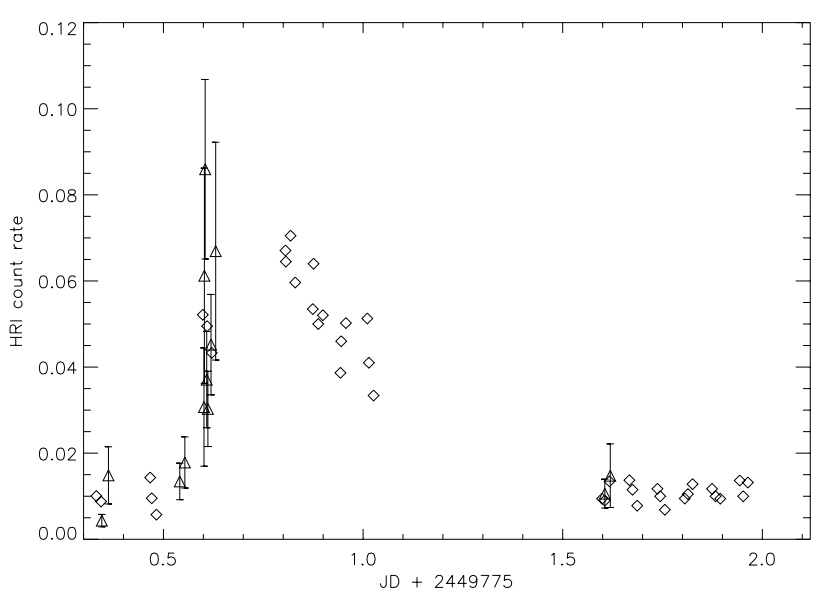

Fig. 3. Superposition of ROSAT HRI light curve of $\sigma$ Ori E (triangles, cf., 2) and the ROSAT PSPC light curve of $\lambda$ Eri (open diamonds, cf., Smith et al. 1993). Note the morphological similarity between the two light curves in the rise phase of the flare.

\subsection{Phasing of the $X$-ray emission and of the $X$-ray flare}

The rotation period of $\sigma$ Ori $\mathrm{E}$ is well known. Using the ephemeris $E=24442778.819+n \times 1.190815$ days, we can assign a phase to each measured data point. The choice of phase is such that $\phi=0$ corresponds to the phase with maximum absorption in the $u$-band (Hesser et al. 1977) which corresponds to the magnetic null phase (Landstreet \& Borra 1978). The corresponding plot is shown in Fig. 4. We have distinguished between flare data points (diamonds) and other data (triangles). Data points taken from an earlier HRI observation of $\sigma$ Ori $\mathrm{E}$ in September 1992, that was already used by Berghöfer \& Schmitt (1994), are represented by asterisks. As is clear from Fig. 4, except for the flare described earlier, no rotational variability of $\sigma$ Ori $\mathrm{E}$ can be recognized. This is in marked contrast to the case of the magnetic early type $\operatorname{star} \theta^{1}$ Ori $C$, whose $\mathrm{X}$-ray emission is modulated with the rotational period of 15.4 days (Gagné et al. 1997). Donati et al. (2002) explain this variability of $\theta^{1}$ Ori $C$ in the framework of the magnetic confined windshock (MCWS) model of Babel \& Montmerle (1997a,b) where the observed modulation is essentially due to the eclipse of the post-shock region by the star itself. No such modulation is apparent in the phased X-ray light curve of $\sigma$ Ori E.

\subsection{Energetics of the $X$-ray flare}

Clearly, the ROSAT HRI observations did not fully cover the flare on $\sigma$ Ori $\mathrm{E}$, and therefore the energetics of this X-ray flare cannot be directly measured. The observed X-ray light curve can be approximated by a straight line, linearly increasing from $0.008 \mathrm{cts} \mathrm{s}^{-1}$ to $0.06 \mathrm{cts} \mathrm{s}^{-1}$ over a time span of $8640 \mathrm{~s}$ (i.e., 0.1 days). We specifically note that the flare observations consist of three time intervals: during a first time interval lasting $1163 \mathrm{~s} 15$ photons were recorded (the 2 light curve data points in Fig. 2 at 0.54 days); during a second time interval yielding only $105 \mathrm{~s}$ of exposure 3 photons were recorded but the bulk of the flare increase occurred in the third time interval 


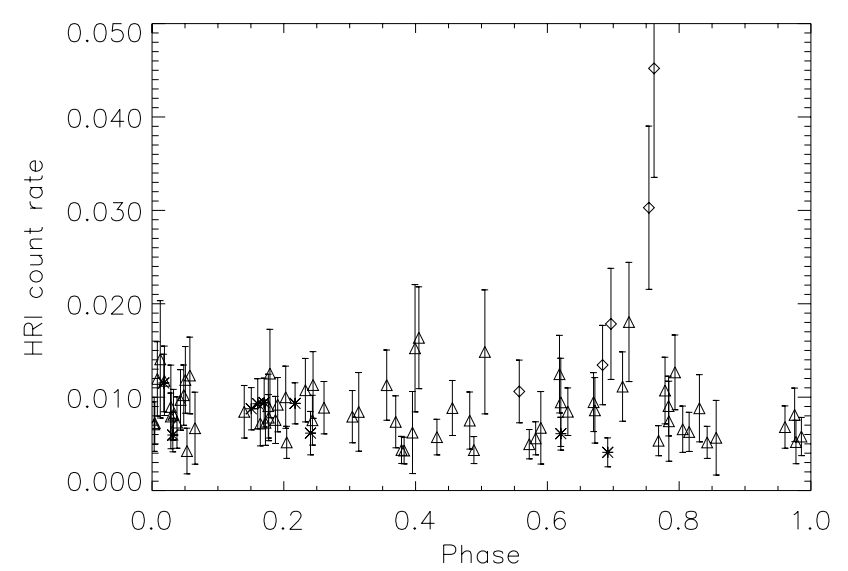

Fig. 4. Phased ROSAT HRI light curve of $\sigma$ Ori E; note that the high data points(diamonds) are due to the flare shown in Fig. 2. While in new (triangles) and old data points (asterisks) no phase dependence of the $\mathrm{X}$-ray emission is detectable.

(after 0.60 days in 2) with a duration of $1490.5 \mathrm{~s}$, during which 69 photons were detected (i.e., the data points between 0.60 and 0.62 in Fig. 2); a fourth data interval of $120.5 \mathrm{~s}$ yielded 8 photons (i.e., the large error bar data point at 0.63 days in Fig. 2). The next data were taken one day later with the count rate being back to pre-flare level. Had this whole increase been continuously observed by ROSAT, a total of 225 counts would have resulted from the flaring X-ray emission (assuming a linear increase in X-ray count rate). The observed flare peak was about $0.052 \mathrm{cts} \mathrm{s}^{-1}$, which of course need not be the actual flare peak, and the whole flare decay remained unobserved by ROSAT. Thus, all we can state is that the flare decay time was $24 \mathrm{~h}$ at most. Assuming then that the count rate started to decrease linearly immediately after the observed flare peak to the pre-flare level $24 \mathrm{~h}$ later, we compute a total of 2250 counts attributable to the flare decay and hence a total of 2475 counts to the whole flare. Alternatively, if we assume that flare rise and decay have the same number of counts, the whole flare would have had 450 HRI counts. With a distance of $640 \mathrm{pc}$ towards $\sigma$ OriE (Hunger et al. 1989) and a flux conversion of $2.4 \times 10^{-11} \mathrm{erg} \mathrm{cm}^{-2}$ count $^{-1}$, which is appropriate for one recorded HRI count corresponds to $1.2 \times 10^{33} \mathrm{erg}$ and hence the total flare energy $E_{\text {tot }}$ emitted at X-ray wavelengths should be $5.3 \times 10^{35} \mathrm{erg}$ and $29 \times 10^{35} \mathrm{erg}$ for the two scenarios discussed. These numbers are clearly not mathematically correct lower and upper bounds for the total flare, however, we think that the true value is very likely bracketed by these numbers. This view is supported by an analysis of the $\sigma$ Ori $\mathrm{E}$ flare reported by Pallavicini et al. (2002); from their $\sigma$ Ori E light curve we estimate a total X-ray energy release of $\approx 1.3 \times 10^{36}$ erg using a distance of $640 \mathrm{pc}$ and a (XMM-Newton) flux conversion factor of $5.4 \times 10^{-12} \mathrm{erg} \mathrm{cm}^{-2}$ count $^{-1}$ (Schmitt et al. 2003). These numbers must be lower bounds to the actual flare energetics, since thermal, kinetic and magnetic energy are not included. Although there are flares on cool stars with even larger energies emitted (more than $10^{37} \mathrm{erg}$ for a long-duration flare on the RS CVn binary CF Tuc (Kürster \& Schmitt 1996) and the Algol-type binary $\beta$ Per (Schmitt \& Favata 1999)), the above considerations show that the flare on $\sigma$ Ori $\mathrm{E}$ is still among the most energetic stellar X-ray flares hitherto observed. The observed peak X-ray luminosity was $6.1 \times 10^{31} \mathrm{erg} \mathrm{s}^{-1}$, again reaching values observed in the strongest cool star X-ray sources.

\section{Interpretation and discussion}

\subsection{Unseen companions?}

Pallavicini et al. (2002) attribute a flare observed with XMM-Newton apparently from $\sigma$ Ori $\mathrm{E}$ to an unseen companion. While the stellar cluster around $\sigma$ Ori $\mathrm{AB}$ is indeed very young and young stars are known to be capable of producing strong X-ray flares, $\sigma$ Ori $\mathrm{E}$ most probably does not belong to the $\sigma$ Ori cluster. Hunger et al. (1989) derived fundamental parameters $T_{\text {eff }}$ and $\log g$ for the stars $\sigma$ Ori E and $\sigma$ Ori D, using high resolution CASPEC data. The latter star turned out to lie near the zero age main sequence $(\log g=4.3)$ with a spectroscopic distance $d=370 \mathrm{pc}$, which is in excellent agreement with the Hipparcos distance of $d=352 \mathrm{pc}$ for $\sigma$ Ori AB, while for $\sigma$ Ori $\log g=3.95$ and a distance of $d=640 \mathrm{pc}$ was reported, clearly identifying $\sigma$ Ori $\mathrm{E}$ as a background star. Using evolutionary tracks from Schaller et al. (1992) with $T_{\text {eff }}=22500 \mathrm{~K}$ and $\log g=3.95, \sigma$ Ori E is a $9 M_{\odot}$ star with an age of $17 \mathrm{Myr}$. Thus both, distance and age make a membership to the $\sigma$ Ori cluster unlikely.

The angular resolution of our HRI data is much better compared to the XMM-data and the relative X-ray positions of $\sigma$ Ori $\mathrm{AB}$ and $\mathrm{E}$ agree very well with their relative optical positions ( $\rho_{\text {opt }}=41.36 \operatorname{arcsec}, \rho_{\mathrm{X}}=41.07 \pm 0.27$ arcsec, and position angle $\alpha_{\text {opt }}=61.55 \mathrm{deg}, \alpha_{\mathrm{X}}=61.60 \pm 0.27 \mathrm{deg}$ ). Because of this excellent agreement of X-ray and optical positions, we consider a chance alignment of $\sigma$ Ori $\mathrm{E}$ with a low mass member of the closer $\sigma$ Ori cluster unlikely.

A positional agreement of only 0.3 arcsec translates into a projected distance of $300 \mathrm{AU}$ of a hypothesized companion of $\sigma$ Ori E. Thus $\sigma$ Ori E could be a spectroscopic binary itself. However, in the high-resolution, high-signal-to-noise FEROS spectra $(S N R \approx 250)$ of $\sigma$ Ori E (cf. Reiners et al. 2000) no sign of any companion can be found. We estimate that any G-star hidden in the spectrum must be smaller than about $0.2 R_{\odot}$ with correspondingly larger limits for M-type stars. If an hypothesized companion of $\sigma$ Ori $\mathrm{E}$ is indeed very young (say 2 Myrs), its radius should be far larger and therefore be visible in the spectrum, contrary to what is observed. Further, radial velocity measurements by Groote \& Hunger (1977) show that any $R V$ velocity variations would have to stay below $1 \mathrm{~km} \mathrm{~s}^{-1}$. And finally, absolute flux measurements from UV to IR, taken from a region encompassing a substantial volume around $\sigma$ Ori E, agree very well with the fluxes calculated from appropriate model atmospheres and provide no evidence whatsoever for a hidden companion or a foreground star possibly belonging to the $\sigma$ Ori system. Nevertheless, a, say, $\leq 0.5 M_{\odot}$ main sequence (i.e. "old") star at $\approx 300 \mathrm{AU}$ might be hidden in the spectroscopic and photometric data, and could thus be held responsible for the observed X-ray emission. Such a star, however, would have an age of $\approx 20 \mathrm{Myr}$ rather than $2 \mathrm{Myr}$, and hence be comparable to clusters like IC 2602 
(Randich et al. 1995) or IC 2391 (Patten \& Simon 1993). G and $\mathrm{K}$-type stars in those clusters show X-ray luminosities about a decade lower than observed for $\sigma$ Ori E. Further, the X-ray flux of $\sigma$ Ori $\mathrm{E}$ is quite constant outside the flare, which is rather atypical for a very young late type star. Also, the observed flare energetics are unusual (albeit not unthinkable) for a $20 \mathrm{Myr}$ late type star. Attributing the observed quiescent and flaring X-ray emission from $\sigma$ Ori $\mathrm{E}$ to a physical companion would thus imply a rather unusual companion star; note that $\sigma$ Ori $E$ is already quite unusual by itself. Therefore, we consider this possibility again unlikely. For the following we therefore assume instead that the observed X-rays and flares are produced in the circumstellar environment of $\sigma$ Ori E.

\subsection{Wind-shock $X$-ray emission}

It seems reasonable to compare $\sigma$ Ori $\mathrm{E}$ to other similar stars. As far as the quiescent flux of $\sigma$ Ori $\mathrm{E}$ is concerned, it is comparable to that observed from other early type stars. However, $\sigma$ Ori $\mathrm{E}$ is a chemically peculiar star with a magnetic dipole field of about $10 \mathrm{kG}$ polar field strength. X-ray emission has been observed from two other magnetic stars, i.e., $\theta^{1}$ Ori C, which is much hotter ( $\left.T_{\text {eff }} \approx 45000 \mathrm{~K}\right)$, and IQ Aur which is much cooler $\left(T_{\text {eff }} \approx 15000 \mathrm{~K}\right)$ than $\sigma$ Ori E $\left(T_{\text {eff }}=23500 \mathrm{~K}\right)$. Their X-ray production can be successfully explained by the MCWS model (Babel \& Montmerle 1997b; Donati et al. 2002); in particular, this model provides a natural explanation for the observed periodicity in the X-ray flux of $\theta^{1}$ Ori C. X-ray flaring for B-type stars has been reported only for $\lambda$ Eri (spectral type B2e; Smith et al. 1993), however, $\lambda$ Eri is so far not known to be a magnetic star.

The central feature of the MCWS model of Babel \& Montmerle is the channeling of the stellar wind emanating at high magnetic latitudes by the magnetic field. The magnetic field forces the high-latitude wind into the magnetic equator plane, where the the wind streams from both hemisphere collide. The shocked gas leads to the observed X-ray emission in the post-shock region; the gas eventually cools and forms a thin cooling disk in the magnetic equator plane. This model would appear to provide an adequate description for $\sigma$ Ori $\mathrm{E}$ at first sight, too, however, the constant X-ray flux of $\sigma$ Ori E (cf., 4) has to be contrasted with the periodic variation of $\theta^{1}$ Ori C. In the latter case the phase variation is interpreted by Donati et al. (2002) as a partial eclipse of the X-ray emitting post-shock region near to the stellar surface at a distance of about $1.5 R_{*}$. The magnetic field of $\theta^{1}$ Ori C is much smaller $(\approx 1.1 \mathrm{kG})$ than that of $\sigma$ OriE $(\approx 10 \mathrm{kG})$, while, on the other hand, the wind from $\theta^{1}$ Ori $C$ is much stronger due to the intense $\mathrm{O}$ star radiation field. Consequently, one would expect a much stronger magnetic confinement in the case of $\sigma$ Ori E, the cooling disk should be denser and extend further out (up to $6 R_{*}$ as observed). Therefore, $\mathrm{X}$-ray absorption at magnetic pole phases with the disk viewed face-on might be expected. The magnetic geometry of $\sigma$ OriE ( $i=54 \mathrm{deg}, \beta=67 \mathrm{deg}$ ) implies that twice during each rotation cycle the disk is viewed edge-on, when X-ray emission should be at maximum, while at least at one phase $(\Phi=0.73)$ the disk is viewed face-on, where X-ray emission should be at a minimum. Such a variation is clearly not observed (cf., 4), and the model successfully explaining the phase variation of the X-ray flux of $\theta^{1}$ Ori C does not appear to be applicable for the case of $\sigma$ Ori E.

What is the reason for the failure of this model describing well the X-ray production in a hotter as well as in a cooler magnetic star? The mass loss of the three stars considered, i.e., IQ Aur, $\sigma$ OriE, and $\theta^{1}$ Ori $\mathrm{C}$ increases from about $10^{-11} M_{\odot} \mathrm{y}^{-1}$ over some $10^{-10} M_{\odot} \mathrm{y}^{-1}$ to about some $10^{-7} M_{\odot} \mathrm{y}^{-1}$, respectively. The low mass loss rate for IQ Aur is the result of non-solar abundances in the wind. Helium (because it is partly neutral) will decouple already in the photosphere, and thus never leaves the stellar surface. Hydrogen decouples near the stellar surface before reaching escape velocity, and will thus be re-accreted if its velocity is too small to reach the magnetic equator. The He-depletion at the magnetic pole (Babel \& Montmerle 1997a) seems more likely to be an $\mathrm{H}$-enrichment due to the re-accretion of hydrogen falling back to the surface (see Groote 2003). The metals instead receive sufficient momentum from the radiation field to be accelerated against gravity to velocities of more than $\approx 800 \mathrm{~km} \mathrm{~s}^{-1}$, and have therefore sufficient kinetic energy to produce the observed $\mathrm{X}$-ray emission. In the case of the $\mathrm{O}$ star wind of $\theta^{1}$ Ori C, radiation pressure on the driving metals is sufficient to accelerate both metals and the passive plasma, which is coupled to the metals through Coulomb forces, to nearly twice the escape velocity $\left(\approx 2500 \mathrm{~km} \mathrm{~s}^{-1}\right)$. In the transition region between massive $\mathrm{O}$ star winds, where metals, hydrogen, and helium are fully coupled, and the pure metallic winds of the cool B stars with essentially no coupling between metals and hydrogen and helium, the mass loss rate decreases with decreasing effective temperature and decreasing wind velocities. Hunger \& Groote (1997) first proposed that the He-enrichment in the atmospheres of He-variable stars may be the result of He-decoupling in the winds of these stars. After decoupling, helium will be re-accreted to form He-rich spots on the stellar surface. Later they extended the idea to He-weak stars (Hunger \& Groote 1999; see also Groote 2003), where hydrogen is also decoupled in the wind and may be re-accreted at the magnetic poles. Such a decoupling of passive elements seems inevitable if wind velocities drop below escape velocity. The coupling becomes weaker with the decrease of density with increasing distance from the star. Once helium (or hydrogen) is decoupled, the remaining wind particles may be efficiently accelerated further because of the substantial decrease in mass left for acceleration. In the case of $\sigma$ Ori E the initial wind velocities are probably low, additionally depending on magnetic latitude, and are perhaps of the order of only $200-300 \mathrm{~km} \mathrm{~s}^{-1}$. Therefore, heating in a post-shock region may be insufficient to produce any significant X-ray emission, so that other heating processes are required. Since no phase variation is observed (Berghöfer \& Schmitt 1994, and this work), we conclude that the source of the observed X-rays is not occulted either by the star nor by the disk, and must therefore be located outside the closed loops of the magnetosphere. This assumption also fits the finding that wind absorption seen in the resonance lines of C IV and Si IV does not vary with phase either (Groote \& Hunger 1997; Smith \& Groote 2001). Groote \& Hunger considered an 
expanding corona outside a wind-fed magnetosphere, which releases matter more or less regularly from the corotating clouds.

\subsection{Size and location of the flaring region}

In view of the above considerations it is likely that also the site of the X-ray flare is located rather far away from the stellar surface. Unfortunately, the essential light curve parameters such as peak flux and decay time are only rather indirectly available from the ROSAT data (see above), and the flare temperature is unknown. For the following we adopt an ad hoc temperature of $10^{7} \mathrm{~K}$, which is a typical temperature for cool star flares. Fortunately, the cooling function of optically thin plasma is rather insensitive to the precise value of temperature in that range. If we further adopt the observed peak X-ray flux of $6.1 \times 10^{31} \mathrm{erg} \mathrm{s}^{-1}$ as the actual peak flux, we compute a minimal emission measure of $E M_{\min } \approx 10^{54} \mathrm{~cm}^{-3}$. As to the decay time, $\tau_{\text {decay }}$, it could be as long as $\tau_{\text {decay }} \approx 40 \mathrm{ks}$ if indeed the same morphology as for the flare on $\lambda$ Eri applies, but also much shorter. If we adopt $\tau_{\text {decay }}=40 \mathrm{ks}$ and further assume that the flare cools only through radiative cooling we can compute a characteristic electron density $N_{\mathrm{e}}$ from the relation

$N_{\mathrm{e}}=\frac{3 k T}{P(T) \tau_{\text {decay }}}$

where $k$ denotes Boltzmann's constant and $P(T)$ the plasma cooling function. For $T \approx 10^{7} \mathrm{~K}$, we find $P(T) \approx$ $10^{-23} \mathrm{erg} \mathrm{cm}^{3} \mathrm{~s}^{-1}$ and hence $N_{\mathrm{e}} \approx 10^{10} \mathrm{~cm}^{-3}$. We consider this a minimal plasma density, since both higher temperatures (which are usually observed in cool star flares) and shorter decay times (40 ks is close to the maximally possible) will lead to higher densities. Some questions remain about the correct cooling function $P(T)$; the values used refer to a fully ionized plasma with cosmic abundances in collisional equilibrium, and these assumptions may not fully apply. Adopting then $N_{\mathrm{e}}=10^{10} \mathrm{~cm}^{-3}$ and $E M_{\text {min }}=10^{54} \mathrm{~cm}^{-3}$, we compute the flaring volume $V_{\text {flare }}=10^{34} \mathrm{~cm}^{3}$, which corresponds to a sphere with radius $1.3 \times 10^{11} \mathrm{~cm}$ or $0.4 R_{*}$. Thus the flaring region is indeed small compared to the star itself, and its presumed distance from the stellar surface. We emphasize that other cooling mechanisms will imply even larger densities and correspondingly smaller volumes. Assuming a field scale size of $1 R_{*}$, the magnetic field strength at $6 R_{*}$ will be about $50 \mathrm{G}$ yielding a pressure of somewhat below $100 \mathrm{dyn}^{-2}$; the thermal pressure of plasma with the above assumed temperatures and densities is about $30 \mathrm{dyn} \mathrm{cm}^{-2}$, i.e., of the same order. Calculating the speed $v_{\text {wind }}$, that a plasma with density of $N_{\mathrm{e}}=10^{10} \mathrm{~cm}^{-3}$ must have to produce a ram pressure of the same magnitude, results in a value of $v_{\text {wind }} \approx 10^{8} \mathrm{~cm} \mathrm{~s}^{-1}$, which would be a plausible wind speed.

Assuming a plasma "cloud" $6 R_{*}$ away from the surface with a thermal pressure of $30 \mathrm{dyn} \mathrm{cm}^{-2}$ is not unreasonable. Groote \& Hunger (1982) derive an electron densitiy of $\approx 10^{12} \mathrm{~cm}^{-3}$ at temperatures $\approx 10^{4} \mathrm{~K}$, yielding thermal pressures of the same order as the hot X-ray emitting gas. Also, the assumption of magnetic reconnection is very plausible. Adopting the above derived values for $V_{\text {flare }}$ and $E_{\text {tot }}$ we can compute the total reconnected field component $B_{\text {recon, }}$ which is found to be $60 \mathrm{G}$, i.e., again of the same order as the (vacuum) dipole field. Thus, a magnetic reconnection scenario requires field strengths and pressures which are entirely plausible.

\subsection{Heating}

The most commonly adopted heating process for single $\mathrm{O}$ and B stars are shocks formed by instabilities in the radiatively driven winds of these stars. Porter \& Skouza (1999) used $1 \mathrm{D}$ hydrodynamic calculations to model the case of $\sigma$ Ori E. According to Porter \& Skouza (1999) hydrogen decouples to form shell-like structures which are then shocked and become the source of the observed X-rays. Krtička \& Kubát (2001) used 2D hydrodynamic calculations (albeit not fully self-consistent, and neglecting magnetic fields and rotation), and found that decoupling of helium is possible in B3 stars when modeling the outflow as a four component gas. Similarly, their models showed decoupling in B5 stars in the context of a 3 component gas. In both cases heating processes are important for the remaining wind component, and temperatures of several $10^{6} \mathrm{~K}$ may be found at larger distances. In the case of magnetic stars the wind will be slowed down additionally by the magnetic latitude dependent channeling of the magnetic field, and their results may also be valid for stars with somewhat higher temperatures. For the case of $\sigma$ Ori $\mathrm{E}$ the heating of the ionic component would then occur outside the closed magnetic field lines in an approximately radial magnetic field line configuration, forming a corona as already assumed by Havnes \& Goertz (1984) and GH1. In that case only a small part of the spherical X-ray emitting region with a diameter in excess of $6 R_{*}$ will be occulted by the dense gas ring. This view is also supported by VLBI observations of $\sigma$ Ori E presented by Phillips \& Lestrade (1989), who find a scale size of 6 to $10 R_{*}$ for the radio emitting regions, once the new (larger) distance towards $\sigma$ Ori $\mathrm{E}$ is taken into account (see also Hunger et al. 1990). Such heating processes will not be present in a star like $\theta^{1}$ Ori $\mathrm{C}$ whose wind is dense and whose wind components are all well coupled. Within the context of such a scenario a plausible explanation for the observed X-ray flare(s) can thus be found. The permanently ongoing filling of the ring/clouds by the wind will increase the plasma ram pressure, while the magnetic pressure stays constant. Once the ram pressure exceeds the magnetic pressure, matter cannot remain confined by the field, and must be released from the magnetosphere. The reconnection of magnetic field lines occurring during this release then provides the additional heating with ensuing X-ray emission. GH1 estimated the time required to refill the clouds using the mass loss and found a time scale of the order of about a month.

\section{Summary and outlook}

The discovery of a X-ray flaring in the Bp-type star $\sigma$ Ori E with a well measured magnetic field of $10 \mathrm{kG}$ provides strong evidence for magnetic field related heating in the magnetosphere of $\sigma$ Ori E. The fact that yet another flare on $\sigma$ Ori $\mathrm{E}$ has been observed with XMM-Newton (Pallavicini 2002) indicates that such flares are not that rare and may occur more or 
less regularly at least on $\sigma$ Ori E. The other early type star with a reported flare is the Be-type star $\lambda$ Eri. Given the similarity of stellar parameters of $\sigma$ Ori $\mathrm{E}$ and $\lambda$ Eri and their winds, one wonders about possible similarities in the physical processes encountered in both types of stars.

Is there then a connection between $\mathrm{Bp}$ and $\mathrm{Be}$ stars? Magnetic fields have already been introduced as a possible source for some of the observed features of Be-type stars and the formation of disks in presence of a magnetic field has been analyzed by Cassinelli et al. (2002) and Maheswaran (2003). We argue here that magnetic fields actually provide the physical link between the two classes of objects. The interaction of wind, magnetic field and rotation is likely responsible for the helium enrichment in the surface spots of early Bp stars. These stars exhibit strong $(B>1 \mathrm{kG})$ magnetic fields but weak stellar winds allowing substantial magnetic confinement (see ud-Doula \& Owocki 2002). Magnetic field strengths, however, should vary considerably from star to star, in particular if the fields are fossil. What would a star look like with only weak magnetic fields present $(50-300 \mathrm{G})$ and a comparable wind as observed for $\sigma$ Ori E? Clearly in this case one would expect the wind to dominate over the magnetic field and align it radially at larger stellar distances as shown by MHD simulations of udDoula \& Owocki (2002). As a consequence there are (nearly) no closed field lines, the wind can flow out freely unhindered by the field, no disk will be formed, and the magnetic field might escape detection. If the wind is not constant (we deal with decreasing wind at decreasing metallicity), we expect the closing of more and more loops when the wind becomes weaker, and therefore the eventual formation of a disk.

The most relevant physical parameters are then the relative strengths of wind and magnetic field (i.e., ram pressure and magnetic pressure) and the value $\beta$ of the magnetic inclination, i.e., the angle between rotation and magnetic field axis, assuming here that the large scale field is dipolar (which is not a necessary condition). Depending on the radiation field, fractionation will occur in the wind for helium (and possibly for hydrogen). Whenever particles of the passive elements do not reach escape velocity, they still have to follow open magnetic field lines, but once left to gravity they will fall back to the stellar surface, mainly along the same field lines. Hunger \& Groote (1999) therefore argued that fractionation is the source of helium enrichment in He-strong stars. The helium enrichment will increase the mass to be accelerated by the driving metals, whose abundance will on the other hand be reduced, leading to a decrease in wind velocity, thus increasing the reaccretion process, which in turn lowers the wind velocity even further. Eventually magnetic field lines reconnect and a disk is formed. On the other hand, once closed loops are present, helium particles will acquire sufficient velocity (although below escape velocity) to reach the disk. Thus the process may be reversed and the helium enrichment is abolished. Alternatively, the helium layer on top of photosphere may become unstable, thus leading to mixing in the photosphere and restoration of normal surface abundances. Once these are restored, the wind strengthens again, the matter in the disk is blown away, and magnetic reconnection is largely inhibited. Thus the proposed scenario, although derived from Bp star modeling, may explain the hitherto little understood temporary appearance of circumstellar disks in Be-type stars, and thus Be-type stars can be regarded as the weak field brothers of the Bp-type stars with small magnetic inclinations.

Weak magnetic fields have already been proposed to explain the higher rotational velocities of Be stars as compared to normal B stars. Stępień (2002) attributes the larger rotational velocities of Be stars to spin up in their pre-main sequence phase and shows that magnetic fields above $400 \mathrm{G}$ lead to slow down, while small magnetic fields between 40 and $400 \mathrm{G}$ lead to an increase in rotational velocity. We then expect at least some helium enrichment also in Be stars, especially prior to the shell phase; such an He-enrichment of $N_{\mathrm{He}} / N_{\mathrm{H}} \approx 0.2$ was indeed determined for the Be star 60 Cygni (Koubský et al. 2000).

The time interval between two shell phases should strongly depend on the polar wind velocity. If this scenario is correct, $\mathrm{X}$-ray flaring should be a common feature of both $\mathrm{Bp}$ and $\mathrm{Be}$ stars. Flares are expected to predominantly occur after magnetic field line reconnection but before or during shell formation. The flare in $\lambda$ Eri might be interpreted as an indication of such an event, since two weeks after the flare there was no $\mathrm{H}_{\alpha}$ emission observed (Smith et al. 1993). At least at that time there had been no evidence for a disk around $\lambda$ Eri.

Acknowledgements. We would like to thank M.A. Smith for a careful reading of our manuscript and extremely helpful comments concerning Be stars, as well as our referee Dr. M. Gagné whose criticisms helped to substantially improve our manuscript.

\section{References}

Babel, J., \& Montmerle, T. 1997a, A\&A, 323, 121

Babel, J., \& Montmerle, T. 1997b, ApJ, 485, L29

Berghöfer, T. W., \& Schmitt, J. H. M. M. 1994, A\&A, 290, 435

Berghöfer, T. W., \& Schmitt, J. H. M. M. 1994, Ap\&SS, 221, 309

Berghöfer, T. W., \& Schmitt, J. H. M. M. 1994, Science, 265, 1689

Berghöfer, T. W., \& Schmitt, J. H. M. M. 1995, Adv. Space Res., 16, 163

Cassinelli, J. P., Brown, J. C., Maheswaran, M., Miller, N. A., \& Telfer, D. C. 2002, ApJ, 578, 951

Cohen, D. H., Cassinelli, J. P., \& MacFarlane, J. J. 1997, ApJ, 487, 867

Donati, J.-F., Babel, J., Harries, T. J., et al. 2002, MNRAS, 333, 55

Drake, S. A., Abbot, D. C., Bastian, D. S., et al. 1987, ApJ, 322, 902

Gagné, M., Caillault, J.-P., Stauffer, J. R., \& Linsky, J. L. 1997, ApJ, 478, L78

Groote, D. 2003, in Magnetic fields in O, B and A stars: Origin and connection to pulsation, rotation and mass loss, ed. L. A. Balona, H. Henrichs, \& T. Medupe, ASP Conf. Ser., CS-305

Groote, D., \& Hunger, K. 1976, A\&A, 52, 303

Groote, D., \& Hunger, K. 1977 A\&A, 56, 129

Groote, D., \& Hunger, K. 1982, A\&A, 116, 64 (GH1)

Groote, D., \& Hunger, K. 1997, A\&A, 319, 250

Havnes, O., \& Goertz, C. K. 1984, A\&A, 138, 421

Hesser, J. E., Moreno, H., \& Ugarte, P. 1977, ApJ, 216, L31

Hunger, K., \& Groote, D. 1999, A\&A, 351, 554

Hunger, K., Heber, U., \& Groote, D. 1989, A\&A, 224, 57

Hunger, K., Heber, U., \& Groote, D. 1990, in Properties of hot luminous stars, ed. Garmany, Proc. First Boulder-Munich Workshop, 307 
Kürster, M., \& Schmitt, J. H. M. M. 1996, A\&A, 311, 211

Krtička, J., \& Kubát, J. 2001, A\&A, 369, 222

Koubský, P., Harmanec, P., Hubert, A. M., et al. 2000, A\&A, 356, 913

Landstreet, J. D., \& Borra, E. F. 1978, ApJ, 224, L5

Linsky, J. L. 1985, Sol. Phys., 100, 333

Lucy, L. B. 1982, ApJ, 255, 286

Maheswaran, M. 2003, ApJ, 592, 1156

Pallavicini, R., Golub, L., Rosner, R., et al. 1981, ApJ, 248, 279

Pallavicini, R., Sanz-Forcada, J., \& Franciosini, E. 2002, High Resolution X-ray Spectroscopy with XMM-Newton and Chandra, Proceedings of the international workshop held at the Mullard Space Science Laboratory of University College London, Holmbury St Mary, Dorking, Surrey, UK, October 24-25, 2002, ed. G. Branduardi-Raymont, published electronically and to be stored on $\mathrm{CD}, \mathrm{E} 29$

Patten, B. M., \& Simon, T. 1993, ApJ, 415, L123

Porter, J. M., \& Skouza, B. A. 1999, A\&A, 344, 205

Phillips, R. B., \& Lestrade, J.-F. 1988, Bull. A.A.S., 20, 728
Randich, S., Schmitt, J. H. M. M., Prosser, C. F., \& Stauffer, J. R. 1995, A\&A, 300, 134

Reiners, A., Stahl, O., Wolf, B., Kaufer, A., \& Rivinius, T. 2000, A\&A, 363, 585

Schmitt, J. H. M. M., \& Favata, F. 1999, Nature, 401, 44

Schaller, G., Schaerer, D., Meynet, G., \& Maeder, A. 1992, A\&AS, 96, 269

Schmitt, J. H. M. M., Fleming, T. A., \& Giampapa, M. S. 1995, ApJ, 450, 392

Schmitt, J. H. M. M., Ness, J.-U., \& Franco, G. 2003, A\&A, 412, 849

Schmitt, J. H. M. M. 1997, A\&A, 318, 215

Schmitt, J. H. M. M., \& Liefke, C. 2004, A\&A, 417, 651

Shore, S. N., \& Brown, D. N. 1990, ApJ, 365, 665

Smith, M. A., Grady, C. A., Peters, G. J., \& Feigelson, E. D. 1993, ApJ, 409, L49

Smith, M. A., \& Groote, D. 2001, A\&A, 372, 20

Stępień, K. 2002, A\&A, 383, 21

ud-Doula, A., \& Owocki, S. P. 2002, ApJ, 576, 413 